D. C. Griffin

Department of Physica, Rollins College

Winter Park, Florida 32783

and Physica Diviaion, Oak Ridge National Laboratory

Oak Ridge, Tennessee 37831

Robert D. Cowan

Log Alamog National Laboratory

Los Alamos, New Mexico 87545

M. S. Pindzola

Department of Physics, Auburn University

Auburn, Alabama 36849

and Physics Division, Oak Ridge National Laboratory

Oak Ridge, Tennessee 37831

\title{
INTRODUCTION
}

58 years ago, Fermil employed the Thomag-Fermi (TF) elatiatical model of the atom to predict a collapes of the $4 f$ orbital for atomic numbers between 55 and 60 , and thereby explained the formation of the first rare-earth series of elemente. In 1941, Maria Mayer ${ }^{2}$ used the same model to predict the onset of a second rare-earth series with the orbital collapse of the $5 f$ electron for atomic numbers between 86 and 91 . She also showed that the abrupt changes in the character of these electrona can be explained in terms of the f-electron effective potential which, in theee regione of the periodic table, consigts of two wells eeparated by a positive centrifugal barrier.

Since Mayer's claseical paper, the regulte of a number of more complete calculations uaing the Thomas-Fermi-Dirac (TFD) model, ${ }^{3}$ as well as the Belf-consistent Hartree-Fock-Slater, ${ }^{4}$ Hartree plus statistical exchange scheme $(\mathrm{HX})^{5}$ and Hartree-Fock (HF) ${ }^{6}$ methods have bean employed to study the variation of $d-, f$ - and even g-electron wave functions within the excited etates of neutral atoms. In the next section of this paper, we employ the results of the more recent self-consigtentfield calculations to consider the now well known changea in radial wave functions for $d$ electrons which occir preceding tise onet of the transition series of elements, and for electrong preceding the onset of the lanthanide and actinide series. The sensitivity of the radial wave functions to variations in the effective polential is discussed, and the large variation of the radial wave functiong between the LS terms of 
certain types of excited configurations in these regions of the periodic ayatem le analyzed.

The changes in the $d$ - and $\mathrm{f}$-radial wave functions along isoelectronic and leonuclear eequences are much more gradual than the sudden contractions of the wave functions that occur within neutral atomic opecies. Neverthelese, euch variatione may lead to large term-dependent effecte in certain excited configurations which are important in electronimpact excitation, a well as photoabeorption. The excitation of innerthell electrons followed by autoionization can in some cases dominate electron-impect ionization crose sections, $7,8,9$ This is especially true in epecles with intermediate to heavy atomic mase where term-dependent effects may aleo be important. In the third eection of this paper, several examples of electron-impact ionization, where the indirect mechanism is dominant and term dependence significantly affects the excitation of the inner-ehell electron, are explained by analyzing the effective potentiale for the excited elecirons in the intermediate autoionizing etates.

Finally, potentlal barriers can lead to significant term-dependent effects in the continuum which play an important role, not only in photoionization, but aleo direct electron-impact ionization. In the fourth section, we consider eeveral examples where such effecta are essential to the accurate determination of both single and double electron-impact ionization cross sections.

THE DOUBLE-WELL EFFECTIVE POTENTIAL AND WAVE FUNCTION COLLAPSE

Near the beginning of the rare-earth aeries of elements the excited $f$ electrong undergo abrupt changes in binding encrgy. This is ahown graphically in Fig. 1 in terme of the effective quantum number $n^{*}$, which

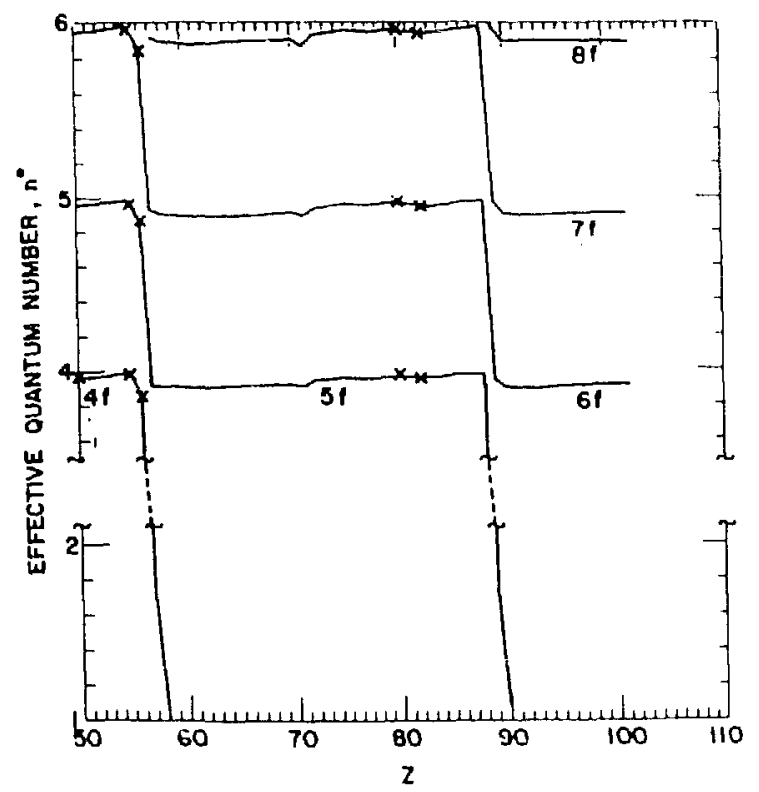

Fig. 1. Effective quantum numbers for $f$ electrons as a function of atomic number from Ref. 5. Curvea, theoretical values calculated using HX binding energieg; $x$, experimental values. 
for neutral atume is derined by the equation:

$$
n^{*}=E_{8}^{-1 / 2} \text {, }
$$

where $E_{\theta}$ is the binding energy of the electron in rydberge. These sudden changes are accompanied by a corrosponding change in the nature of the $f$ electrons consisting of contractions of the wave functions to smaller radii. In order to underetand these effects it is useful to introduce an effective central potential, so that the radial differential equation can be written ag:

$$
\left[-\frac{d^{2}}{d r^{2}}+V_{n f f}(r)\right] P_{n}(r)=B_{n} t P_{n}(r) \text {. }
$$

where the effective potential, $V_{\text {a }} f(r)$, and the eigenvalue, $E_{n} l$, are in rydberg units. If one employe the Hartree-Fock method, $V_{\text {eff }}(r)$ is deined by the equation:

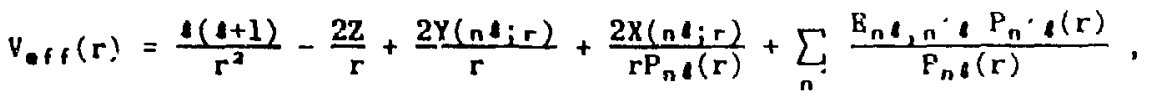

where we use a notation similar to Fiacher'e. 10 In Eq. (3), $Z$ is the atomic number; $2 Y(n z ; r) / r$ is simply the direct potential when the subshell Is aingly occupied, as it is for the cases of interest here; $2 X(n l ; r) / r$ is the exchange function; and $E_{n} l_{n}$, is a Lagrangian multiplier used to force orthogonality between radial wave functione with equal values of but different $n$. This function is well defined for nodeless radial wave functions, ouch as $P_{t r}(r)$, and in other caseg a simple interpolation may be used to prevent aingularities in a plot of $V_{\text {ef }}(r)$.

For electrons in an open subshell, $V_{e f f}(r)$ will be different for the different LS terms, resulting in term-dependent radial wave functions. We will shortly consider cases for which euch variation among the terms is quite aizable; however, for many other cases, $V_{e f f}(r)$ and $P_{n} l(r)$ are nearly the same for all terms of a configuration, and we may then employ a configuration-average Hartree-Fock (CAHF) method for which the direct polential and the exchange function are the game for all terms. When this is the case, it is possible to employ various approximationg to the CAHF method, in which the Lagrangian multipliers, $E_{n} \ell_{n}^{\prime}$, are set to zero and the exchange function is replaced by some approximate, local exchange potential. The best known of these are the Hertree-Fock-Slater method, 11 in which the exchange function is replaced by Slater's free-electron gas exchange potential, and the modified HFS potential by Herman and Skillman. 12 In addition, there is the Hartree plus atatistical exchange 8cheme 13 which incorporates an improved free-electron gas exchange potential and yields one-electron energies which agree quite well with those obtained from the CAHF method.

With today's high speed computers and improved numerical techniques, the HF or CAHF problem can be solved routinely and the various locel epproximations to the Hartree-Fock method are no longer widely used. However, most of the work done on wave function collapse in the neutral atoms was performed with one of these methoda, and therefore they are included here. Furthermore, it is important to note that the HF equations are not solved in the form given in Eq. (2), but rather in their proper inhomogeneous form. The HF form of $V_{e}(f, r)$ is used only as a descriptive device in explaining the nature of wave function collapse.

In hydrogen, the effective potential reduces to the form: 


$$
V_{\text {.ff }}(r)=\frac{\Delta(l+1)}{r^{2}}-\frac{2}{r}
$$

With $t=3$, for $f$ electrons, this is positive for $r<6$ (Bohr unite), and beyond this there exista a shallow fotential well with a mimimum at $r=12$. In miulti-electron atoms, the core-electron wave functions do not extend appreciably into this outer region, and therefore, the potential for $r>6$ is nearly hydrogenic. However, for large 2 , a second inner well develope at amall radii where the effective nuclear charge ia quite large. These two wolle are geparated by a positive centrifugal barrier, which if high enough, resilts in the existence of two quasi-independent sets of negative energy levele. Since the inner well is very narrow as compared to the outer hydrogenic region, the energy levele ageociated with this well are very widely epaced, and until $Z$ becomes quite large, the lowest energy eigenvalue for the gysten is that of the outer well. Thus the binding energy for an $f$ electron wiil be nearly equal to the hydrogenic value and $n$ en. As 2 continues to increase, we reach a point where the inner well is aufficiently deep and wide that the inner-well level becomes the lowest one, and we then see an abrupt increase in the binding energy and an agsociated decrease in $n^{*}$.

The f-electron wave function has appreciable amplitude in only one of the two well regions, depending on whether the eigenvalue corresponde to an energy level of the inner or outer well. These two alternatives are shown in Fig. 2 for the $4 f$ wave function in neutral 58 Ba and 5 La. The $4 f$ wave function in $B a$ lies almost entirely in the cuter-well hydrogenic region, the eigenvalue is nearly equal to that of hydrogen and $n^{*}=3.8$. However, in La the inner well is oufficiently deep and wide that the $4 \mathrm{f}$ wave function has collapsed completely into the

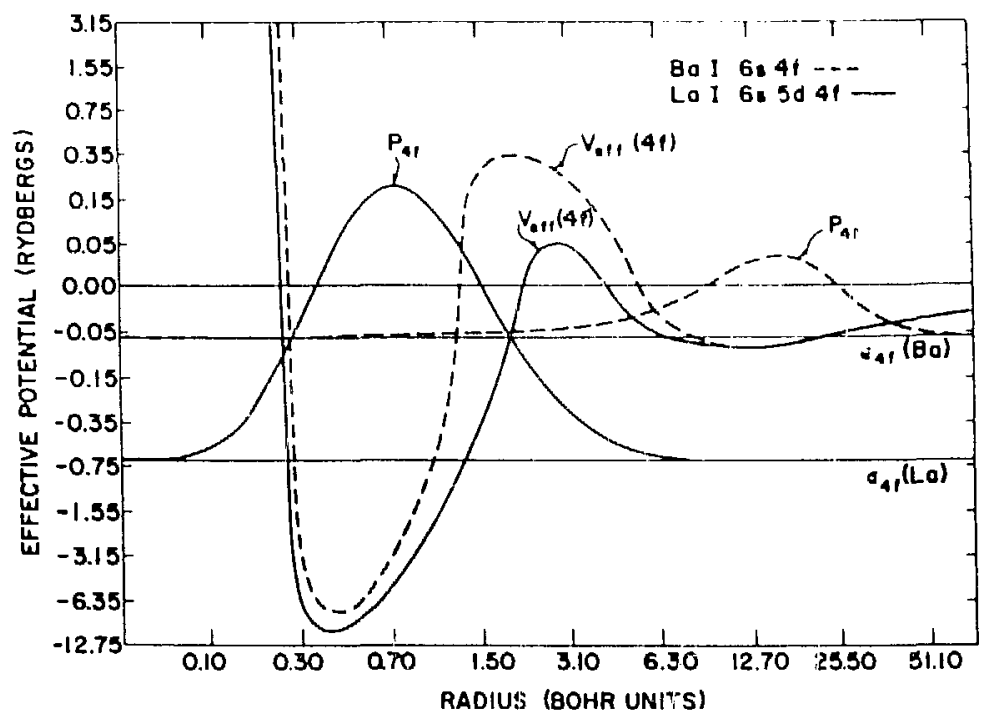

Fig. 2 Plot of the effective potentials and radial wave functions for the $4 \mathrm{f}$ electron in neutral Ba and La calculated using the HX approximation (Ref. 5). Nonlinear scales are used for both the effective potential and the radius. The radial wave functiona are plotted on a linear scale the zero of which lies at the corresponding eigenvalue, noted in the figure by $\varepsilon_{4}$. 
inner-well region with a correaponding abrupt decrease in $n^{*}$.

At the aame point where the $4 f$ wave function collapses into the inner well, the firat antinode for the $5 f$ wave function moveg abruptly from the outer region into the inner-well and barrier regiona and becomes quite omall in magnitude. This to illustrated in Fig. 3 where, in La, only the second antinode of the $5 f$ wave function has any appreciable amplitude, and it liea in the outer-hydrogenic region where ita shapo resembles that of the $4 f$ wave function in $\mathrm{Ba}$. Thus the quantum defect $\left(n-n^{*}\right)$ changes abruptly from a value close to zero to a value of nearly one in going from $2=56$ to $2=57$. More generally, if for a given atomic number the wave functione for the $m$ lowest-lying $n f$ aubshells are in the inner well, then all excited nf wave functiong have $m$ antinodes with relatively emall amplitudes in the inner-woll or potential-barrier region, and therefore $n-m-3$ antinodes with much larger amplitudes in the outer-well, hydrogenic region. This implies an effective quantum number $n{ }^{*} n-m$, in agreement wilh the trends for $f$ electrons shown in Fig. 1.

It is posaible that an inner-and outer-well level might have equal energies. In such a case, the wave function would have large amplitudes in both welle and a minimum in the barrier region. For a fixed potential such as TF or TFD, such a situation will exist at some, in general non-integral, value of 2 . However, for neutral atoma with an excited electron for which 123, where the barrier height is quite large, such a gituation will be ungtable when cne uses a self-consistent-lield model. If a emall portion of charge is trangferred from the outer to the inner well during the iterative procese, the other electrons become more completely shielded from the nucleus and move to larger $r$; this in turn causes the excited electron to be lese well shielded and it moves further toward the inner well; the inner well becomes deeper and wider and its level finally drope below the outer well level causing the excited electron to collapse completely into the inner region. This argument, which explains the

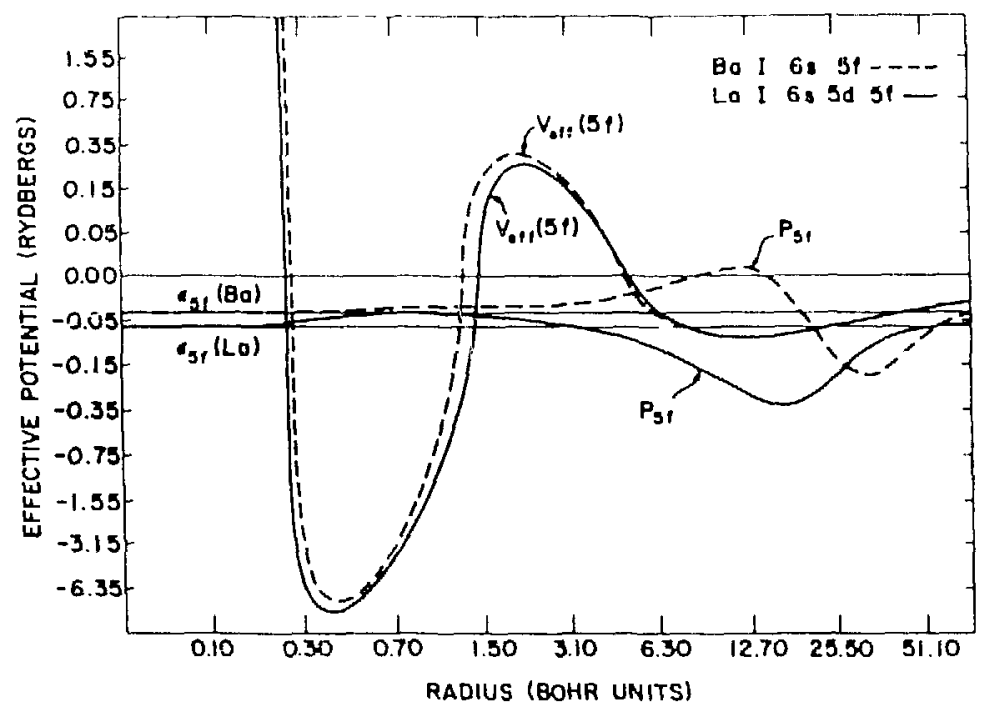

Fig. 3. Plot of the effective potentials and radial wave functiong for the $5 f$ electron in neutral Ba and La calculated uaing th.s HX approximation (Ref. 5). Further deocription is given under Fig. 2. 
abrupt nature of the contraction for $f$ electrong in neutral epeciea, is valid only when the barrler height is large and we can correctly dlecuse two quasi-independent sets of energy levela. Ag we shall see, when the barrier height is omall, there are cases where even a self-conaiatent procedure will yleld appreciable amplitudes in each of the two regions.

Now let us consider the elluation for d electrons. On the bagis of the TF potential, Mayer ${ }^{2}$ noted that double welle with positive potential barriers cannot occur for $d$ electrons, and thus concluded that abrupt whve function and binding-energy changes should not occur for d electrons. However, Latter's subsequent calculations, ${ }^{3}$ which employed both the TF and TFD potentials, corrected to have the proper $-2 / F$ long range behavior, showed changes in energy similar to those for f electrons, although somewhat more gradual. Furthermore, Rau and Fano 4 pointed out that potential barriers do exist for d electrone when one uses a reasonebly accurate potential, euch as HFS with a tail cut-off at $-2 / r .12$

The plot of effective quentum numbers for d electrone calculated uaing the HX potential is shown in Fig. 4. As can be seen, the chenges in $n^{*}$ with $Z$ are atill pronounced, although somewhat lese abrupt than in the cas of $f$ electrons. The potential barriers for d electrons, when they do exist, are much more tenuous. For $t=2$, the hydrogenic well beging at $r=3$ and has its minimum at $r=6$. Thus, in a multi-electron atom, the core-electron wave functions will tend to overlap into the hydrogenic region, reducing the possibility of barrier formation. The barrier will be the most pronounced when the core electrons are the most compact, auch as in the case of a closed-shell core like $K\left(3 p^{6} 3 d\right)$ where $\langle r\rangle_{3 p}=1.43$.

The effective potentials and radial wave functiong for the $3 d$ electron in $K$ and $\mathrm{Ca}$ are shown in Fig. 5. The effective potential for $K$ does indeed have a well defined barrier region, although its maximum is actually below zero. The $3 d$ wave function lies predominately in the

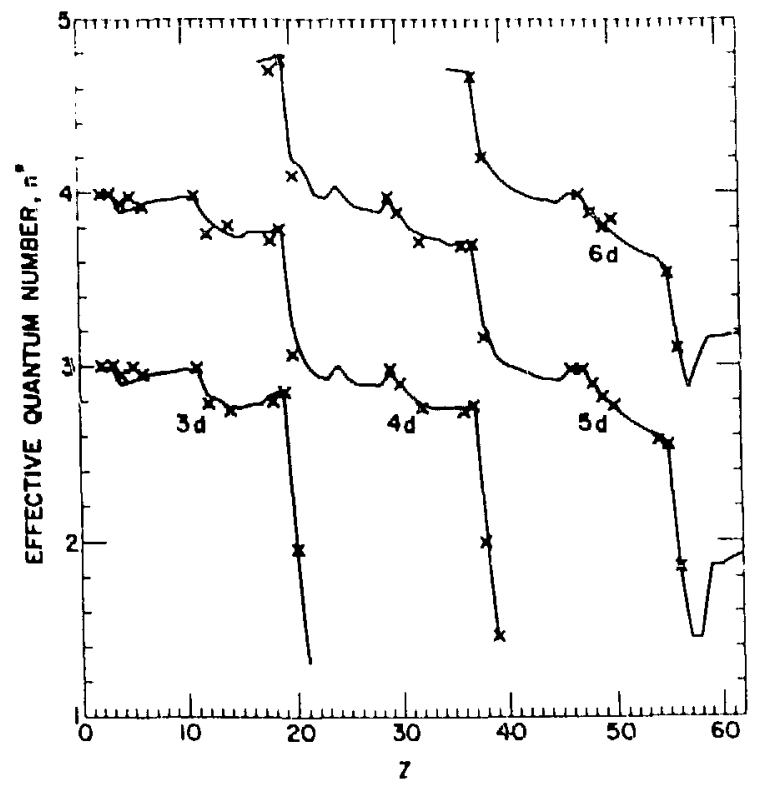

Fig. 4. Effective quantum numbers for d electrons as a function of atomic number from Ref. 5. The notation is the same as in Fig. 1 . 


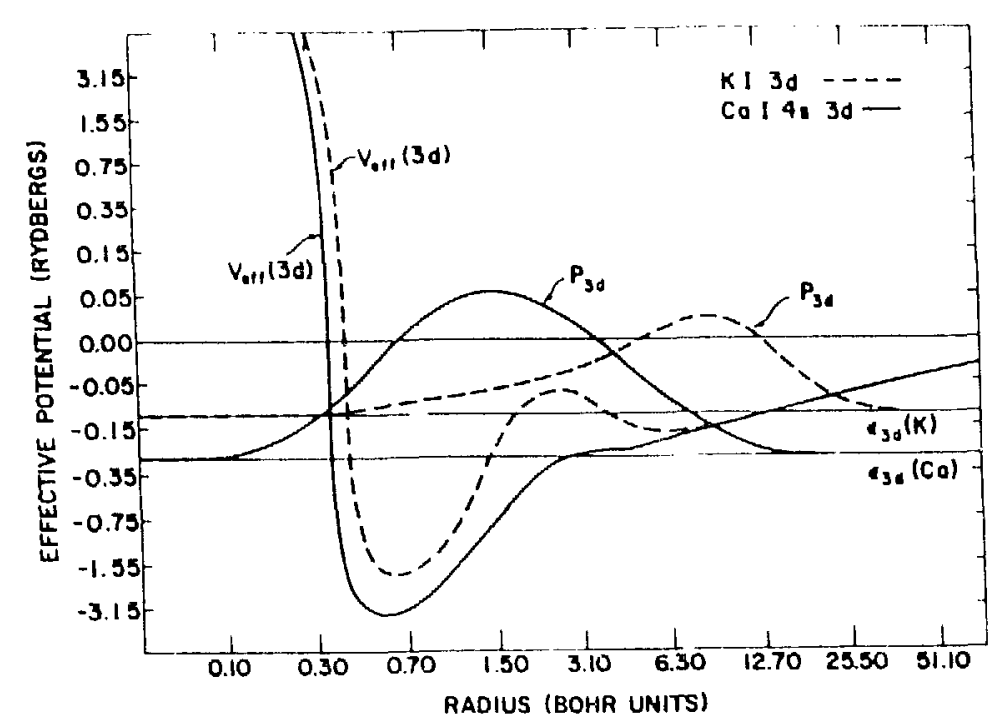

Fig. 5. Plot of the effective potentiala and radial wave functions for the $3 d$ electron in neutral $K$ and $C a$ calculated using the $\mathrm{HX}$ approximation (Ref 5). Further description is given under Fig. 2.

outer well; however, it does have a noticeable overlap with the core region. In the next element $\mathrm{Ca}\left(3 p^{6} 4 \mathrm{~s} 3 d\right)$, the $4 \mathrm{~s}$ electron extends far into the outer-well region and provides very poor shielding for the added unit of nuclear charge. As a result, the barrier is wiped out, and the $3 d$ wave function moves much further into the core region with the accompanying large change in $n^{*}$ displayed in Fig. 4. The situation with the excited d electrons for which $n \approx 4$, is similar to that for the $n f$ electrons already discussed, although again less dramatic.

Since the potential barriers for d-electron effective potentiala are low in height, or not even present, the outer well regions are much less hydrogenic than they are for $f$ electrons. This is the reason why, in Fig. 4, the $\mathrm{n}^{*}$ curves are not nearly as flat between drops, the values of $n^{*}$ are not as close to integer values and the changes in $n^{*}$ are not as close to unity as in the case of the $f$ electrons shown in Fig. 1 . This is especially true for high $\mathrm{Z}$ where the core-electron wave functions are lese compact, and do not tend to provide the shielding necessary for the formation of well defined barriers. In generall, the sudden variations in d-lectron wave functions are much more a function of the detailed nature of the ahell structure of the atom, and therefore much more Bensitive to the core-electron configuration. Furthermore, predicted locatione of $d$-wave function collapes within the periodic syetem are much more dependent on the central-field model employed.

All of the above discussion pertains only to neutral atoms; however, some of the more interesting physics associated with these centrifugal barrier effects occurs in ionized epecies. The effective potential for a multiply-ionized atom, can be written in the form:

$$
V_{\text {aff }}(r)=\frac{1(t+1)}{r^{2}}-\frac{2 Z_{a f f}}{r}
$$

where $Z_{\text {aff }}$ is the effective nuclear charge. For an excited electron which remains outaide the core electrons in a $\mathrm{Z}-\mathrm{N}$ ionized atom, $\mathrm{Z}$. ff 
reduces to the hydrogenic-like value $\mathrm{Z}-\mathrm{N}+1$, where $\mathrm{N}$ is the number of

electrons. We see from Eq. (5) that the radlue where the outer, hydrogenic-like well begine is inversely proportional to the value of the effective nuclear charge, and thus it decreases with ionization stage. Therefore, the core-electron wave functions, which have about the eame radii in iong as in neutrals, have much larger overlapg with the outer well, oven for ex. We then would expect much amaller barrier heights in ions, and these shculd decreses and become lese important as a function of lonization atage.

In Fig. 6, we show the $\mathrm{HX}$ effective potential and radial wove function for the $4 f$ electron in $\mathrm{Ba}^{+}\left(5 \mathrm{p}^{\circ} 4 f\right) .14$ AB can be seen, the effective potential is much lese pronounced than in the case of neutral Ba shown in Fig. 2, and in fact, has a top which is negative. As a result, the $4 f$ wave function is bimodal in shape with an appreciable amplitude in both the inner- and cuter-well regione. Nevertheleas, the largest portion of the amplitude of the wave function remains outgide, and the quantum defect is relatively smali $(\alpha, 32)$.

As $n$ increases, the barrier has gradually lese effect on the nf wave Punctiong, and their firat antinodea penetrate deeper into the inner-well region. 15 This leade to some rather unusual properties in this $n f$ Rydberg series. For example, the value of the quantum defect increases toward unity with increasing $n, 5$ more rapidly at first (for $n=5, n-n^{*}=.65$ ), and then very gradually, reaching a value of .85 by $n=10$. Such variations are so unusual that, for a time, they were suspected to be the result of misinterpretation of the observed spectra.14,16 The behavior of these nf wave Punctions also leads to some unusual featurea in the absorption spectrum of $\mathrm{Ba}^{+}$from the long lived $5 \mathrm{~d}^{2} \mathrm{D}$ states, 17 as discussed by Connerade. 15

As we would expect from the above considerations, the d-electron

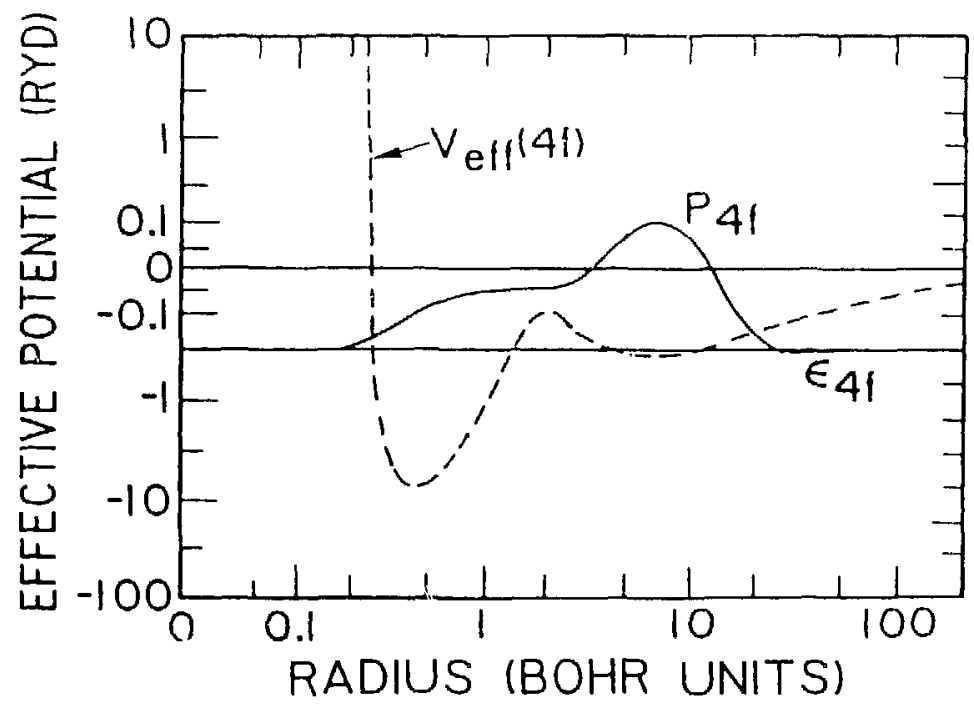

Fig. 6. Plot of the effective potential and radial wave function for the $4 f$ electron in Bat calculated uaing the HX approximation (Rer. 14). Further description is given under Fig, 2. 
and $\mathrm{P}$-electron orbitale collapee gradually as a function of ionization stage as compared to their variation as a function of $\mathrm{z}$ for the noutral atome. Neverthelese, these contractions can have important effects on the atomic atructure of excited states involving $d$ and $f$ electrons. A discussion of the effects of such wave function variations for d electrons along isoelectronic eequencer is given, for example, in Ref. 18. The contraction of $f$ electrons as a Punction of ionization stage will be discuseed in detail in the next section.

We now consider cases in which there is gignificant term dependence, or variation of the radial orbitals among the terms of a configuration. When this occura, the CAHF method or the various local, configuration-average approximations mentioned in the laet section no longer provide an accurate description for the system. Such termdopendent effects were first atudied by the Hartrees 19 in a calculation of the $282 p$ configuration, and a discussion of the results for the $282 p{ }^{3} P$ and $l_{P}$ terms in $B e$ is given by Fischer. 10 In this example, the $2 s-2 p$ exchange interaction in $B$ causes the $2 p{ }^{3} \mathrm{p}$ orbital to be slighily contracted $(\langle r\rangle=2.9)$, while the $2 p{ }^{1} \mathrm{P}$ orbital is repelled to much larger radii $(\langle r\rangle=5.0) .10$

These term-dependent effecte are even more pronounced in configurations of the type $p^{3} d$ near the beginning of the tranaition series, and $d 9_{f}$ near the beginning of the rare-earth series of elements. In order to understand this, we consider the energy expressions for the ip terms of these two configurations:

$$
\begin{aligned}
E\left(p^{5} d^{1} F\right)=E_{a v a}\left(p^{3} d\right)-0.200 F^{2}(p d) & +1.267 G^{2}(p d)-0.043 G^{3}(p d) \\
E\left(d^{9} f^{1} P\right)=E_{a v a}\left(d^{9} f\right)-0.229 F^{2}(d f) & -0.095 F^{4}(d f)+1.957 G^{1}(d f) \\
& -0.019 G^{3}(d f)-0.021 G^{3}(d f),
\end{aligned}
$$

where $F^{k}(t, 1$,$) and G^{k}(1,1$,$) are the Slater parameters for the direct and$ exchange electrostatic interactions, respectively; and the spin-orbit interaction terms are not included. The other terms of these configurations have coefficiente for the electrostatic interactions of comparable magnitude, excent for the coefficienta of the dipole exchange integrals, $\mathrm{G}^{1}$, which are small. Therefore, if we apply the variational principle to these energy expreseions to obtain the term-dependent HF equations, the resulting exchange functione are quite different for the ' $P$ terms a compared to all other terms of the configuration; however, the potential terms and exchange functions for the other terms are well approximated by the CAHF method.

The large positive dipole exchange interactions within the expressions for $V_{\text {eff }}(r)$ of the ' $P$ terms can produce pronounced doublewell effective potentials for the excited $d$ and $f$ electrons in such configurations. The sensitivity of the radial wavefunctions to these barriere may, in turn, lead to large term-dependent variations which have significant effects on a number of important atomic properties. Extensive calculations for configurations of the type $p^{3} d$ have been performed by Hansen. 20 One of the most axtreme examples of these effects for $d^{\circ} f$ configurations occurs in the excited configuration $4 d^{9} 5 g^{2} 5 p^{8} 6 s^{2} 4 f$ of neutral Ba. In Fig. 7 we show the $4 f$ electron HF effective potentials and radial wave functions for this case. 21 The configuration average (CA) effective potential, which provides an accurate representation of the potential for the nine terms other than $1 P$, has a very amall barrier and a deep inner well. The large difference between this potential and that Bhown in Fig. 2 for the $4 d^{10} 58^{2} 5 p^{6} 684 f^{f}$ configuration of $B a$ is due to the reduction in shielding when an electron is promoted from the closed $4 \mathrm{~d}$ subshell to the 68 subshell. The $C A$ if wave function has collapsed into 


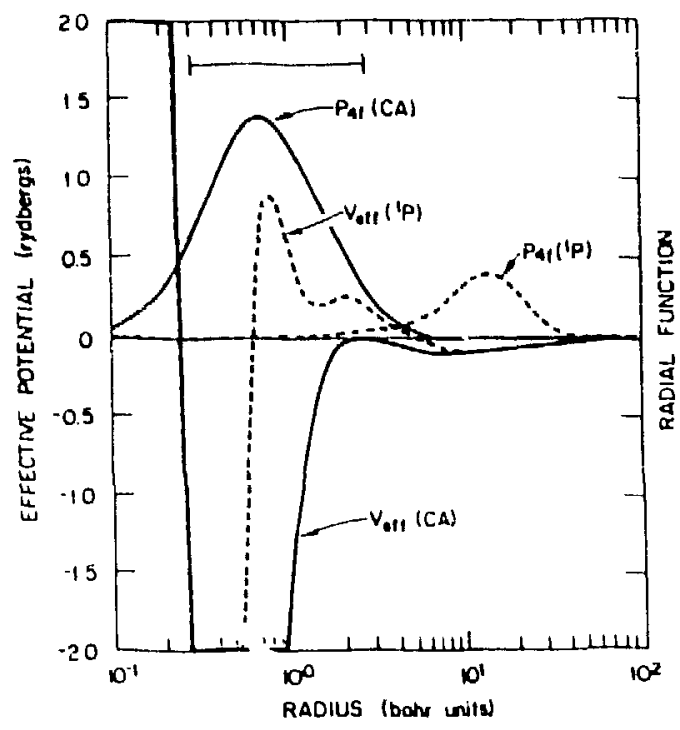

Fig. 7. Plot of the HF $4 f_{C A}$ (solid curves) and $4 f^{1} \mathrm{P}$ (dotted curver) effective potentiale and radial wave functione for the the $4 d^{9} 58^{2} 5 p^{6} 6 g^{2} 4 f$ configuration in neutral Ba (Ref. 21). A logarithmic acale is used for the radius. The bar indicates the range of radii over which the second antinode of the $4 d$ radial wave function occurs.

the inner region, has a mean radius of 1.17 and a large overlap with the 4d electrons within the core. On the other hand, the 'P effective potential has two distinct positive barriera; the large inner one ia due to the positive exchange term, while the smaller outer one is due to the centrifugal term. However, contrary to the frozen-core term-dependent HF effective potential of Wendin and Starace, 22 the above polential, determined from a fully-relaxed, self-consiatent HF calculation, does not go below zero in the region between the two barriers. These barriers cause the ' $\mathrm{p}$ 4 $\mathrm{f}$ wave function to remain in the outer-well region where it: closely resembles a $4 \mathrm{f}$ hydrogenic wave function, has a mean radius of 17.5 and an extremely small overlap with the $4 \mathrm{~d}$ radial wave function.

When one uses the $4 f_{\mathrm{CA}}$ wave function to calculate the position of the ${ }^{2} P$ term it is found to lie above the $4 d^{9} 58^{2} 5 p^{6} 6 \theta^{2}$ ionization threshold because of the very large value of $G^{1}\left(4 d, 4 f_{C A}\right)$. However, as firat pointed out by Hansen et. al., 23 when one uses the proper $4 f^{\prime} \mathrm{P}$ wave function, the $4 d^{\circ} 4 f^{\prime} \mathrm{P}$ term lies below the $4 d^{\circ}$ ionization limit and has a binding energy close to that of a $4 f$ hydrogenic electron.

Since all the nf ' $P$ wave functions in the $4 d^{9} 5 g^{2} 5 p^{6} 6 g^{2} n f$ configurations of neutral $B a$ lie in the outer hydrogenic region, and thus have very small overlaps with the $4 \mathrm{~d}$ wave function, the photo-abeorption transitions $4 d^{\prime a} \rightarrow 4 d^{9} n f^{\prime} P$ will have very little oscillator otrength. The oscillator atrength is, instead, tranaferred to the continuum trangition $4 d^{10} \rightarrow 4 d^{\prime} \varepsilon f^{\prime} P$, which is the cause of the well known giant resonance in the photoionization cross section of neutral Ba. This phenomenon will be discussed in detail by other authors in this volume, and therefore will not be pursued any further here.

In considering LS term dependence, it is important to note that one can approximate these effects by using $C A$ radial wave functions and 
performing a configuration-interaction calculation which includes the other nembers of the Rydberg eeries. For example, Younger 24 has performed euch a calculation for $X^{\circ}{ }^{\circ}\left(4 d^{\circ} 4 f\right)$ and was able to approximate the term-dependent if ' $P$ wave function from en expension of $C A$ wave functions of the form:

$$
P_{4}\left({ }^{1} P\right) \otimes c_{1} P_{4 f}(C A)+c_{3} P_{3 f}(C A)+c_{3} P_{6 f}(C A),
$$

where $c_{1}, c_{2}$ and $c_{3}$ are the mixing coefficiente in the configurationinteraction calculation. A similar calculation has been performed for $\mathrm{K}^{+}\left(3 \mathrm{p}^{3} 3 \mathrm{~d}\right.$ ' $\left.\mathrm{P}\right), 14$ but the Rydberg aeries nembers through $\mathrm{n}=11$ had to be included before resonable agroement was obtained. Finally Wendin 25 and Wandin and Starace 22 have performed extengive CA, Rydberg gerie日 interaction calculations on the neutral Ba case just considered, in which very large basis sets had to be included.

By Brillouin's theorem, 26 configuration-interaction integrals which connect members of a Rydberg series, like the ones discussed above, will vanish when one usea the proper term-dependent HF wave Punctions. That is, correlations of the type represented by such interactions are already included in the zerc-order, term-dependent wave functions. However, this is not true for $C A$ wave functions. Nevertheless, if a complete set of CA wave functions are employed in a Rydberg series expansion, the resulting wave function must be identical to that obtained from the solution to the term-dependent HF equations. The primary advantage of the $\mathrm{CA}$ approach is that one can include LS term dependence and the effects of the spin-orbit interaction in a single multi-configuration calculation; the primary disadvantage is that many configurations may be required, including continuum states, before an accurate represenlation is obtained.

\section{ELECTRON-IMPACT EXCITATION-AUTOIONIZATION}

The study of electron-impact ionization increases our understanding of collisional dynamice and atomic atructure and has important applications in laboratory and astrophysical plasmas. In the last decade, experinental and theoretical investigations have demonatrated the importance of the indirect mechanism involving the excitation of an inner-shell electron, followed by autoionization, to the total ionization process; in fact, for certain intermediate and heavy atomic sytems in lower atages of ionization, these indirect contributions can completely dominate the ionization cross section. $7,8,9$

The processes we wish to consider her's are:

$$
\begin{aligned}
& e+\Delta q^{+} \rightarrow \Lambda(q+1)++e+e, \\
& e+\Lambda q^{+} \rightarrow\left(\Lambda^{q+}\right)^{*}+e \\
& L_{A}^{(q+1)++e}
\end{aligned}
$$

where $q$ is the charge of the etomic ion; Eq. (9) represents direct ionization, while $E_{q}$. (10) represente the excitation of an inner-ahell electron to an intermediate state, followed by autoionization. The contribution of excitation-autoionization to the ionization crose section ia given, to a very good approximation, by the the excitation crose section times a branching ratio for autoionization versus radiative stabilization. For relatively low stages of ionization, where the autoionizing rate is much higher then the radiative rate, this branching ratio ia normally equal to unity, unless there are selection rules which inhibit 
autoionization from a particular lovel.

When the first atep in Eq. (10) involves an excitation of the form $d^{10} \rightarrow d^{9} f$, then the potential barrier elfocts and the resulting term dependence discussed in the last section become important in the determination of the size and shape of the ionization cross section. One of the best examples of this occurs in the cd isoelectronic Bequence. 27 Energy-level diagrame showing the $4 d^{10} 5 g^{2}$ ground-state configurations and the excited $4 d^{\circ} 5 \theta^{3} 4 f$ conilgurations for the Cd-like

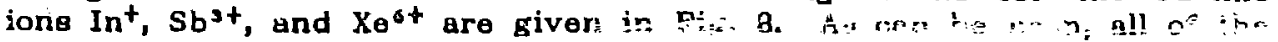
20 levels of $4 d^{9} 5 g^{2} 4 f$ are autoionizing tor the rirsi two ions, while only the $d^{\circ} 5 g^{2} 4 f^{\prime} P_{1}$ level is above the lonization threahold in $X 9^{\circ+}$. The contribution of excitation-autoionization through the $4 d^{\circ} 5 g^{2} 4 f$ configuration in $\mathrm{In}^{+}$is small. This can be understood by studying Fig. 9 where plote of the $4 f$ effective potential and radial wave function for th: ion are shown. The 'P effective potential has hoth $\&$ large potential barrier and positive minimum in the inner well; es a result, the if i $P$ wave function must remain in the outer well-region. However, the $C A$ effective potential, which provides a fairly accurate representation for the other nine terms, has a much emaller barrier and the minimun in the inner well is now negative; neverthelese, the $4 f_{C A}$ wave function atill reaides in the outer region, and is nearly identical to the $4 f^{\prime}$ ' $P$ wave function. Thus the overlep of these wave functions with the $4 d$ radial wave function is minimal, and the collision strength for the $4 d^{10} \rightarrow 4 d^{\circ} 4 \mathrm{f}$ electron impact excitation is extremely small.

As we move elong the isoelectronic sequence, thinge begin to change, and by $\mathrm{Sb}^{3+}$, term dependence sets in, as can be seen by examining Fig. 1G. The increase in nuclear charge nearly wipes out the $C A$ potential barrier, causing the $4 f_{C A}$ wave function to drop into the inner region. However, the effective potential for the 'P term still has

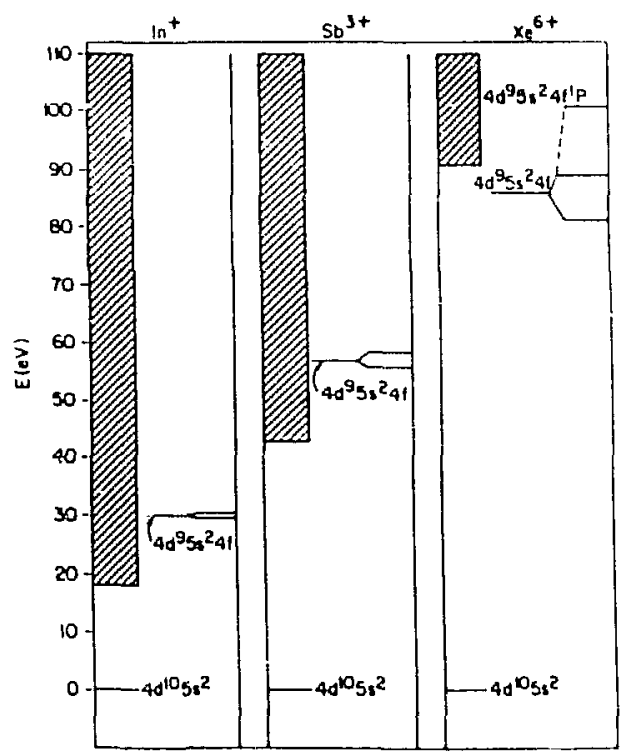

Fig. 8. Energy-level diagrams showing the $4 d^{10} 58^{2}$ ground-gtate configurations, the $4 d^{9} 58^{2} 4 f$ excited configurations and the ionization thresholds of $\mathrm{In}^{+}$, $\mathrm{Sb}^{3+}$, and $\mathrm{Xe}^{6+}$ (Ref. 27). Hatched regions indicate the ionization continuum for the next higher ionization stage. 


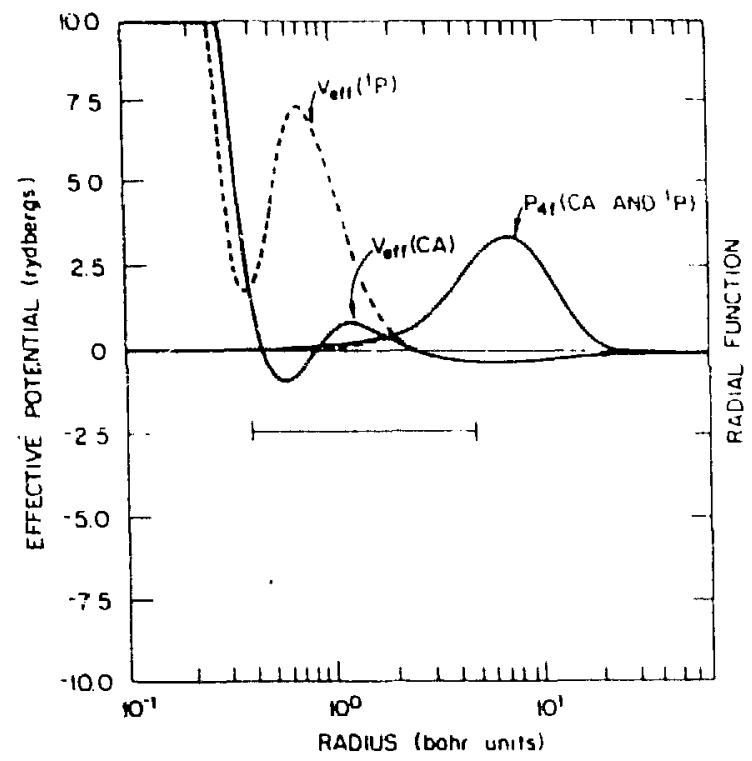

Fig. 9. Plots of the $4 f_{C A}$ and $4 P^{2} P$ effective potentials and radial wave functions fur the $4 d^{\prime} 58^{2} 4 f$ configuration in $\mathrm{In}^{+}$(Ref. 27). The notetion is the same as in Fig. 7.

a large positive oarrier, which forces the $4 f{ }^{1} \mathrm{P}$ radial wave function to remain in the outer region. This has a prorounced effect on the gize and shape of the $4 d^{10} 5 s^{2} \rightarrow 4 d^{9} 5 s^{2} 4 f$ excitation cross section. The size of the dipole-allowed transition to the ' $P_{1}$ level depends on the overlap of the $4 d$ and $4 f{ }^{2} \mathrm{P}$ wave functions which, in this ion, is quite amall. However, the non-dipole excitations to the other 19 levels of $4 d^{9} 5 s^{2} 4 f$ depend primarily on the size of the exchange sontributions to the cross

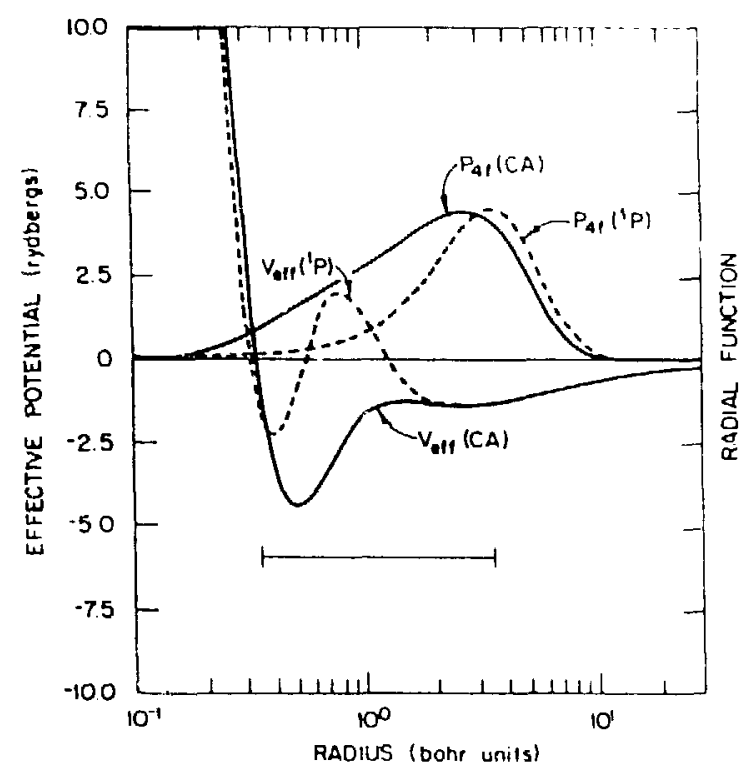

Fig 10. Plots of the $4 \mathrm{f}_{\mathrm{CA}}$ and $4 \mathrm{f}$ ' $\mathrm{P}$ effective potentiale and radial wove function for the $4 d^{9} 5 \theta^{2} 4 f$ configuration in $\mathrm{Sb}^{3+}$ (Ref. 27). The notation is the game as in Fig. 7. 
section, and these contributions, are in turn, a function of the overlap of the $4 \mathrm{~d}$ radial wave function with the outgoing scattered wave, as well as the overlap of the $4 f_{\mathrm{fA}}$ wave function with the incident wave. For electron energies near threshold, the incident wave has its firet node in the berrier region, and thus there is a large overlap between ite firet antinode and the $4 \mathrm{f}_{\mathrm{cA}}$ wave function which has a large amplitude in the inner-well region. However, ae the energy increaseg, the firct node of the incident wave moves further into the inner region and cancellation effecta begin to decrease the magnitude of the collision atrength. Therefore, in $\mathrm{Sb}^{3+}$ we would expect the axcitation crise eection for $4 d^{105 a^{2}} \rightarrow 4 d^{1} 5 a^{2} 4 t$ to be dominated by the non-dipole transitions to the 19 levels other than ${ }^{1} P_{1}$, and on the basis of the above discuesion, its magnitude should fall off rapidly with electron energy.

The theoretical lonization crose section for $\mathrm{Sb}^{3+}$, showing the excitation-autoionization contributiong from both the $4 d^{9} 5 s^{2} 4 f$ and $4 d^{\circ} 5 \theta^{2} 5 f$ configurations is given in Fig. 11 . The $5 f$ configuration is also term dependent and the crose eection is again dominated by the non-dipole transitions. The dipole collision strength has been trangferred to higher members of the Rydberg series, and perhaps into the continuum. Thus, we would expect some of the dipole-allowed transitione to the $4 d^{1} 5 a^{2} n f^{\prime} P_{1}(n>5)$ levels to make measurable contributions to the ionization crose section, and they should be included in a complete calculation. These general concluaione are supported by a racent crosed electron-ion beam experiment performed on $\mathrm{Sb}^{3+}$ at $O \mathrm{ORNL}^{28}$, which shows a large indirect contribution to the ionization cross section of the shape shown in Fig. 11, but with apparent contributions from higher members of the Rydberg series. In ardition, the experimental results show evidence for the added indirect process of resonent recumbination followed by double autoionization, 29 which will not be discussed here. Finally, it should be mentioned that large non-dipole dominated excitation-autoionize.tion contributions are also observed in several members of the $\mathrm{Xe}$ isonuclear sequence in low stages of ionization. 29

Plote of effective potentials and radial wave functions for $\mathrm{Xe}^{6 t}$, the last ion in the Cd sequence to be considered, are shown in Fig. 12. The

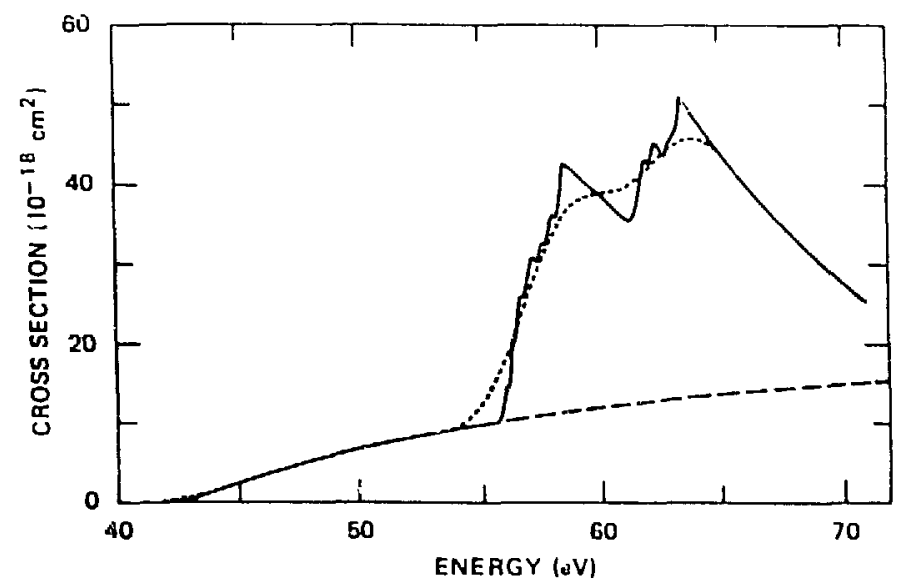

Fig. 11. Ionization crose section for $\mathrm{Sb}^{3+}$ (Ref. 27). Dashed curve, direct-ionization crose section caiculated from the Lolz equation; eolid curve, distorted-wave calculation of the indirect contribution due to $4 d^{10} 58^{2} \rightarrow 4 d^{9} 5 s^{2} n f(n=4,5)$ plua Lotz; dotted curve, solid curve convoluted with a $2-\mathrm{eV}$ Gaussian to aimulate the experimental energy spread. 


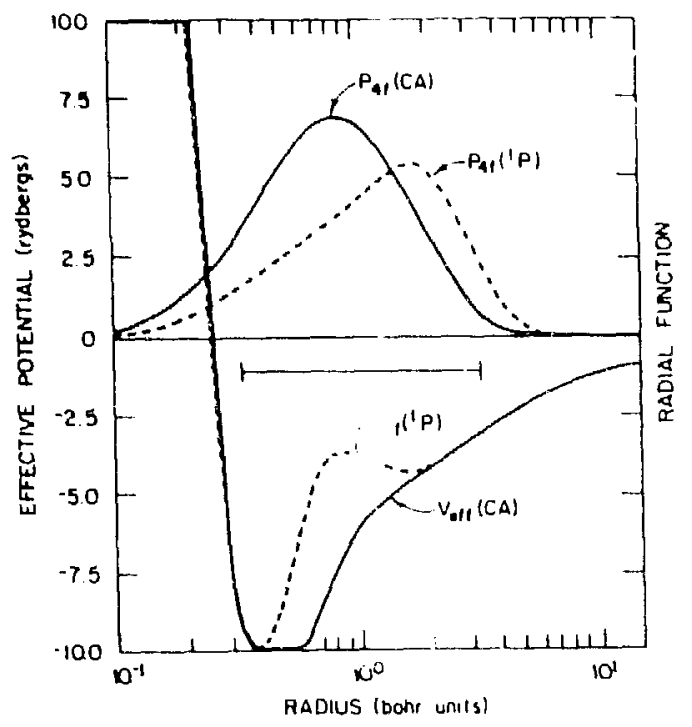

Fig. 12. Plote of the $4 \mathrm{f}_{\mathrm{CA}}$ and $4 \mathrm{f}{ }^{4} \mathrm{P}$ effective potentials and radial wave iunctions for the $4 d^{9} 5 \theta^{2} 4 f$ configuration in $\mathrm{Xe}^{6+}$ (Ref. 27). The notation is the same as in Fig. 7.

additinnal increase in nuclear charge has caused the potential barrier for the ${ }^{1} \mathrm{P}$ effective potential to become negeive and small, and the $4 f{ }^{2} \mathrm{P}$ wave function is on the verge of sliding ccmpletely into the inner-well region. For still higher stages of ionization the barrier will become completely insignificant, the $4 f$ i $P$ wave function will become indistinguishable from the $4 f_{C A}$ wave function, and term-dependent effects will disappear. Nevertheless, for $\mathrm{Xe}^{\text {st }}$, these offects are atill quite important and must be included in order to obtain accurate theoretical values of the ionization cross section. This is illustrated in Fig. 13, where we show the contribution of the $4 d^{10} 5 s^{2} \rightarrow 4 d^{9} 5 g^{2} 4 f^{1} P_{1}$ excitation followed by autoionization to the ionization crose section. It is legitimate to treat the only autoionizing level of the $4 d^{9} 5 s^{2} 4 \mathrm{f}$ configuraiion, ${ }^{1} P_{1}$, eeparately since intermediate-coupling calculations indicate that it is over 99\% pure. Aa can be seen, the distorted-wave calculations show a factor-of-2 reduction in the crose section when the proper if ' $P$ wave function, rather than the $4 f_{C A}$ wave function is used. The very small difference between the term-dependent, distorted-wave results and the results one obtains when the $4 f^{\prime} \mathrm{P}$ wave function is employed in a two-state, close-coupling calculation indicates that it is term dependence, and not continuum coupling, which is important for this case.

The more complete resulis for $\mathrm{Xe}^{6+}$ calculated using the distortedwave method are shown in Fig. 14, along with the croseed electron-ion beam measuremente of Gregory and Crandall. 30 The agreement between experiment and theory is quite good. The $4 d^{9} 58^{3} 4 f^{\prime} p_{1}$, level at $100.4 \mathrm{eV}$ makes the largest contribution to the ionization cross eection. However, the indirect contributions from other levels are also important. The atep at $116.4 \mathrm{eV}$ is due to the $4 \mathrm{~d}^{7} 5 \mathrm{~s}^{2} 5 \mathrm{f}^{1} \mathrm{P}_{1}$ level. The first antinode of the $5 \mathrm{f}$ 'p wave function overlapa more strongly with the second antinode of the $4 d$ wave function than does the first antinode of the $5 \mathrm{f}_{\mathrm{CA}}$ waye function, which has moved further inside. This increases the cross section to the $51^{2} \mathrm{P}$, level when the proper term-dependent radial wave function is used. Thus some of the $4 I^{\prime} \mathrm{P}$ collision strength has been transferred to 5f ' $\mathrm{P}$. Also included in this calculation are the excitation-autoionization 


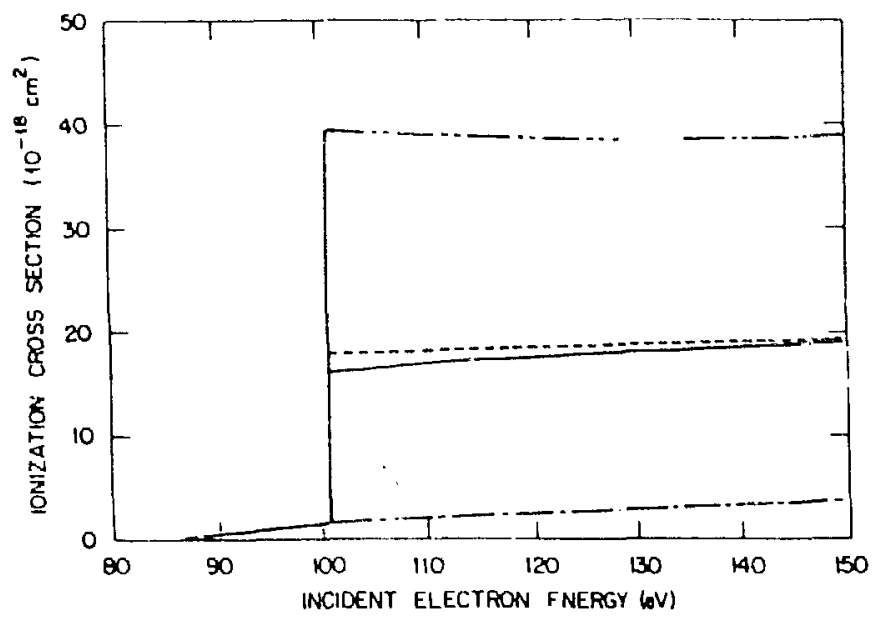

Fig. 13. Ionization cross section for $\mathrm{Xe}^{\text {st }}$ showing the indirect contributions from the $4 d^{9} 58^{2} 4 f^{2} P_{1}$ level (Ref. 27). Dot-dash curve, direct-ionization crose eecion from the Lotz equation; solid curve, term-dependent, close-coupling' calculation for the $4 \mathrm{~d} \rightarrow 4 \mathrm{f}^{1} \mathrm{P}_{1}$ excitation plus Lotz; dotled curve, ter-n-dependent, distorted-wave calculation of the $4 \mathrm{~d} \rightarrow 4 \mathrm{P}$ ' $\mathrm{P}_{1}$ excitation plus Lotz; chain curve, $\mathrm{CA}$, distorted-wave calculation of the $4 \mathrm{~d} \rightarrow 4 f^{\prime} \mathrm{P}_{1}$ excitation plus Lotz.

contributions from the $4 d^{9} 5 g^{2} 5 d$ and $4 d^{9} 5 s^{2} 6 p$ configurations, which show no term dependence. Finally, we include in Fig. 14 the excitation crose section to the lower levels of $4 d^{9} 5 s^{2} 4 f$ (marked $O$ for optical transitions) which are below the ionization threshold and, of course, do not contribute to the ionization cross section.

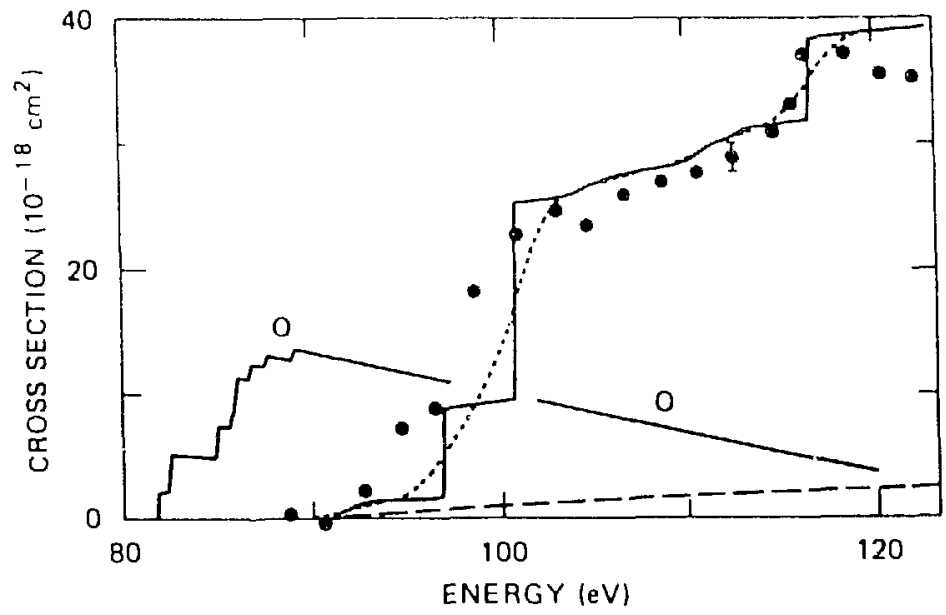

Fig. 14. Ionization crose section for $\mathrm{Xe}^{6+}$ (Ref. 27). Deshed curve, direct-ionization crose section calculated from the Lotz equation; solid curve, distorted-wave calculation of the exciLations $4 \mathrm{~d} \rightarrow 4 \mathrm{f}, 5 \mathrm{f}, 5 \mathrm{~d}, 6 \mathrm{p}$ plus Lotz; dotted curve, eolid curve convoluted with a $2-\mathrm{eV}$ Gaussian to simulate the experimental energy apread; solid curve narked $O$ is the total excitation cross section to non-gutoionizing levels of $4 d^{9} 5 \mathrm{~s}^{2} 4 \mathrm{f}$; filled circles, experimental measurements, Ref. 30. 
Potential-barrier and term-dependent effecta can also be importent for excitation-autoionization tranaitions involving excited d electrons near the beginning of the transition eeries of elements. This is especially true in trangitions of the type:

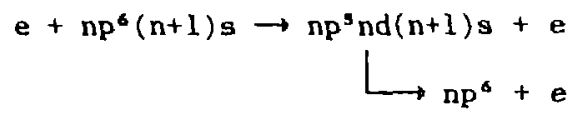

in the singly ionized species $\mathrm{Ca}^{+}, \mathrm{Sr}^{+}$, and $\mathrm{Ba}^{+}$. These are the first ions where excitation-autoionization was found experimentally to dominate the total ionization crose eection. $31,32,33$ Hansen 34,35 first showed that the $n p^{3} n d(n+1)_{8}$ configurations in these ions exhibit pronounced term dependence, and he predicted that this would have a significant effect on the cross section for the $\mathrm{np}^{6} \rightarrow \mathrm{np}$ id excitation. Since then, an R-matrix calculation 36 has demonstrated the importance of continuum coupling in this excitation process, and distorted-wave calculations ${ }^{37}$ have confirmed Hanaen's prediction regarding the aignificance of term dependence. However, to date, no calculation has been reported whirh includes both of tinese effects, and sizable disagreement batween theory and experiment peraiste near the ionization threshold. Theoretical work un these ions, aimed at gaining a better understanding of the combined effecta of term dependence and continuum coupling, is continuing.

\section{DIRECT-IONIZATION CROSS SECTIONS}

Potential-barrier and term-dependent effects in the continuum, whicn have been shown to be of great importar.: in certain photoionization transitione (see for example the review culas by Conneradels and Karaziya ${ }^{38}$ ), may also be significant in direct electron-impact ionization. Distorted-wave calculations have demonetrated that term dependence in the ejected-electron continuum can have a large effect on both the size and shape of single 39,40 and double electron-1inpact ionization crosa sections. 41,42

Let us first consider electron-impact ionization out of the ad subshell within the Xe isonuclear eequence: 41,42

$$
e+X e^{\bar{q}^{+}}\left(4 d^{10} 5 s^{2} 5 p^{6-q}\right) \rightarrow X e^{(q+1)+}\left(4 d^{9} 5 s^{2} 5 p^{6-q}\right)+e+e,
$$

where $\mathrm{q}$ is the ionization stage of the initial ion. For $\mathrm{Xe}^{+}, \mathrm{Xe}^{2+}$, and $\mathrm{Xe}^{3+}$, all states of the configuration $4 \mathrm{~d}^{9} 5 \mathrm{~s}^{2} 5 \mathrm{p}^{6-q}$ are above the first ionization threshold, while for $\mathrm{Xe}^{+t}$, only one third of the states of $4 d^{9} 58^{2} 5 p^{2}$ are autoionizing. Thus for the first three ions, we would expect that ionization out of the $4 d$ subshell should be followed by autoionization and contribute to the double-, rather than the single-, ionization crose section; in the case of $\mathrm{Xe}^{4+}$, assuming a statistical distribution of the collision strength, about one third of the $4 \mathrm{~d}$ ionization crose section will be observed as double ionization.

In all these ions, the $4 \mathrm{~d}$ ionization is dominated by the $4 \mathrm{~d}^{9} \mathrm{kf} \mathrm{f}^{1} \mathrm{P}$ ejected-electron cisannel, and as one would expect from prior discusaion, this channel is highly term dependent. In Fig. 15 we show the plots of the $\mathrm{CA}$ and ${ }^{1} \mathrm{P}$ effective potentials and continuum orbitals for $\mathrm{Xe}^{+}$and $\mathrm{Xe}^{2+}$ for an ejected-electron energy of $1 \mathrm{eV}$. For $\mathrm{Xe}^{+}$the CA effective potential has a small negative barrier, while the ' $P$ effective potential has large positive barrier. As a result, the îrst antinode of the $k f_{C A}$ radial wave function is located in the inner-well region, where it has a large overlap with the second antinode of the $4 d$ orbital; while the firat anti- 
node of $\mathrm{Kf}$ ' $\mathrm{P}$ le primarily in the outer-well region, where it overlaps only weakly with the eecond antinude of the $4 d$ orbital. $A$ atrong ovarlap between the $\mathrm{kf}$ 'P orbital end the $4 d$ orbital will not occur until we reach hisher ojected-electron energies. For $\mathrm{Xe}^{2 t}$, at the oame ejected-electron onargy, the gituation is quite different. With the decreased shielding reaulting from tne removal of another $5 p$ electron, the barrier in the CA offoctvo potentiel has dieappeared and the $k f_{C A}$ has moved far enough into the inner region that wave cancellation has begun to decresse its overlap with the $4 d$ wave function. However, the decrease in the ip potential barrier has caused the kt ip continuum arbital to elide much further into the inner region, where it has a sizable overlap with the eccond antinode of the $4 \mathrm{~d}$ wave function.

On the basis of these simple effective-potential arguments, we can make a number of predictione regarding the 4d ionigation crose section for these two ione. In $\mathrm{Xe}^{+}$, the use of the proper term-dependent orbital, rather than the configuration-average orbital, for the $\mathrm{kf}$ i $\mathrm{P}$ ejected-electron channel should lead to a reduction of the crose section near threshold (i.e., a delayed onset) and, most likely, an overall decrease in the magnitude of the crose section. However, in the case of $\mathrm{Xe}^{2+}$, the 4d ionization crose section calculated with the term-dependent continuum function should be larger then that calculated with the CA orbital, even near threshold.

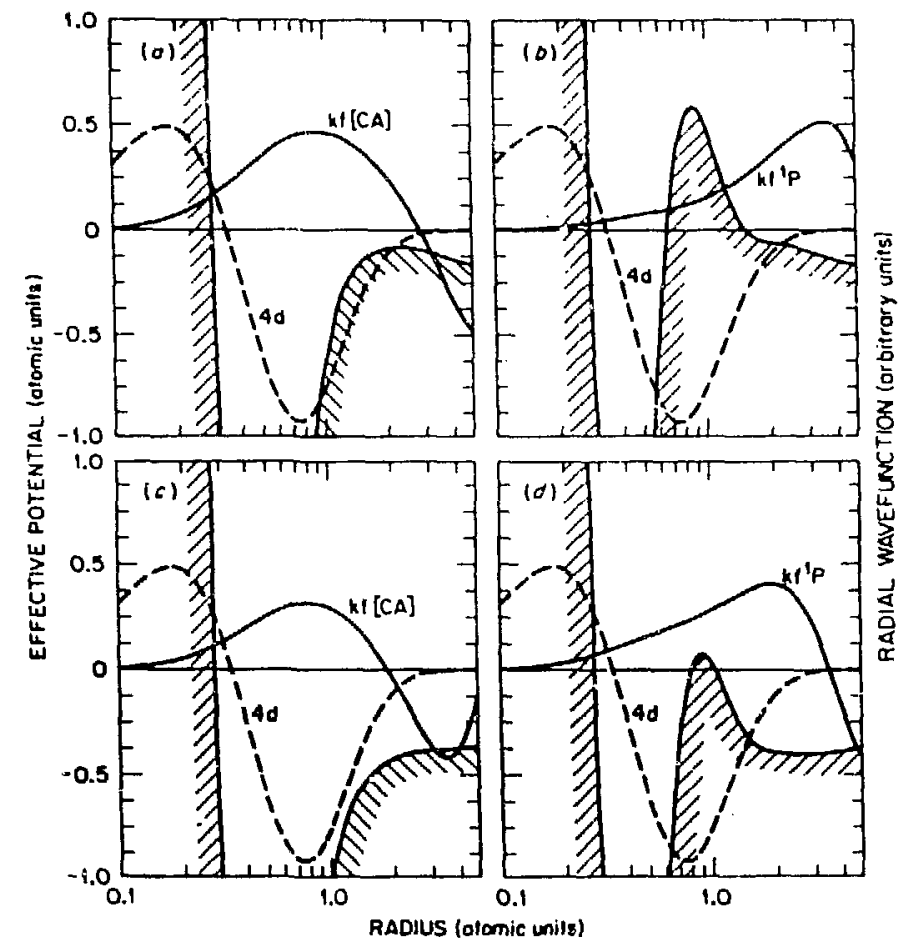

Fig. $15 \mathrm{CA}$ and $\mathrm{P}$ term-dependent offoctive potentials and radial wave functions for the $f$ continuum electron ejected from the 4d subahell in $\mathrm{Xe}^{+},(a)$ and (b), and $\mathrm{Xe}^{2+},(c)$ and (d) (Ref. 42). Hatched solid curves, effective potentials; solid curves, kf sontinuum wave functions at an energy of 1 eV; daehed curves, $4 d$ bound orbital. Note here that the offective potentials are in hartree atomic units, rather than rydbergs. 
The experimental and theoretical results for double ionization in $\mathrm{Xe}^{+}$ through $\mathrm{Xe}^{4+}$ are shown in Fig. 16. The direct double-ionization crose eection was estimated from the binary-encounter approximation of Gryzinski, 43 and as expected, it decreases rupidly with ionization stage. The 4d ionization-autoionization contributions, however, dominate the total double-ionization cross section for all 4 lons, and show significant terendependent effecte. The calculations which employ the kf 'P termdependent continuum functions for the $4 d^{\prime \prime} k f$ ' $P$ ejected-electron channel aleo include an estimate for the effecte of ground-state correlation by iricorporating the important $4 d^{10} \longleftrightarrow 4 d^{\circ} 4 t^{2}$ configuration interaction. The difference botween the $\mathrm{CA}$ and the term-dependent resulte for $\mathrm{X}_{\theta}{ }^{+}$ and $\mathrm{Xe}^{2+}$ is about as expected from the potential-well arguments above and, in each case, the term-dependent results are closer to the experimental measurements. 44 The results for $\mathrm{Xe}^{3+}$ are eimilar to those for $\mathrm{Xe}^{2+}$; however, in all three ions the experimental threshold is below that predicted by theory, In the case of $\mathrm{Xe}^{4+}$, we slso show curves for the 4d lonization cross aections multiplied by the fraction of atates of the configuration $4 d^{9} 5 s^{2} 5 p^{2}$ which autoioniza (1/3). From a compariocn of these scaled curves with the experimental measuremente, it would appear that the collision strength is not distributed statistically.

Recently Younger 40 completed a theoretical study of photoionization and direci electron-impact ionization ou't of the $4 \mathrm{~d}$ subshell for ten ions

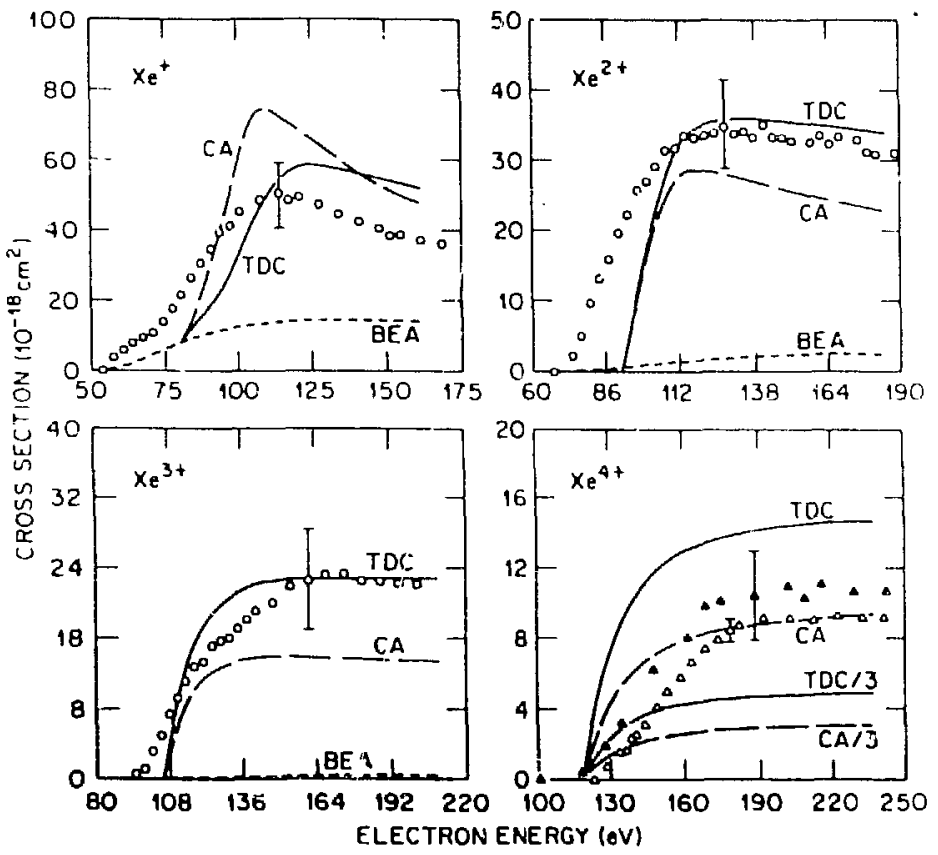

Fig. 16. Electron-impact double-ionization crose sections for (a) $\mathrm{Xe}^{+}$, (b) $\mathrm{Xe}^{2+}$, (c) $\mathrm{Xe}^{3+}$, and (d) $\mathrm{Xe}^{4+}$. Open circles and darkened triangles, experimental data (Reí 44); open triangles, experimental data (Ref 42); short-dashed curves labeled BEA, direst-double ionization uaing the binary-encountor approximation; long-dashed curve labeled $C A, 4 d$ ionization contribution using configuration-average kf radial wave functions plus direct-double ionization; solid curves labeled TDC, 4d ionization contribution using of ${ }^{1} P$ term-dependent radial wave functions with groundstale correlations plus direct-double ionization. 
within the palladium isoelectronic gequence, with ground-gtate configurations $4 \mathrm{~d}^{\prime \circ}$. The crose gections are, as in the cases discussed above, dominated by' the $4 d^{2} \mathrm{kf}$ ' $\mathrm{P}$ channel, and term dependence was found to be aignificant up to a charge etate of +10 . In an earlier calculation, 45 he demonstrated gimilar effecta for electron-impact ionization out of the closed $5 \mathrm{~d}$ subshell in $\mathrm{Hg}^{+}$.

Term dependence in the ejected-electron continuum may aleo be important in electron-impact lonization out of closed $p$ subshells. This was shown in a study of the argon isoelectronic sequence 39 where, for low stages of ionization, the $3 p$ ionization crose section is dominated by the $3 p^{5} \mathrm{kd}$ ip ejected-electron channel. In neutral argon, the term-dependent effective potential for the d continuum electron differa aignificantly from the $\mathrm{CA}$ effectlve potentlal and has a well defined barrier recion. Thua at low ejected-electron energies, the kd ip partial wave, unlike the $k d_{C A}$ wave, will not penetrate the barrier and will have a small overlap with the 3p orbital; however, at some higher ejectedelectron energy the penetration will become appreciable. This behaviour, which is similar to that already discusesed for the $k P{ }^{1} \mathrm{P}$ wave in $\mathrm{Xe}^{+}$, is displajed in Fig. 17 where the phase shift, $\delta$, is plotted as a Punction of ejected-electron energy for the $C A$ and term-dependent kd partial waves. The phase shift for the $\mathrm{kd}_{\mathrm{CA}}$ wave is large at very low energie日, while the kd 'P phase ahift is quite amall until the wave beging to penetrate the barrier at about $4 \mathrm{eV}$.

The ionization crose eection for Ar I calculated uaing the CA and term-dependent kd partial wave for the $3 p^{5} \mathrm{kd} i \mathrm{P}$ ejected-electron channel are shown in Fig. 18. As can be Been, term-dependence is quite important for electron eriergies up to more than three times threehold. Also shown is a term-dependent calculation corrected for the effects of ground-state pair correlation $3 p^{8} \longleftrightarrow 3 p^{4} 3 d^{2}$. Term-dependence enhances the influence of this interaction by increasing the overlap of the kd continuum wave and the $3 d$ correlation orbital. 39 For the higher members

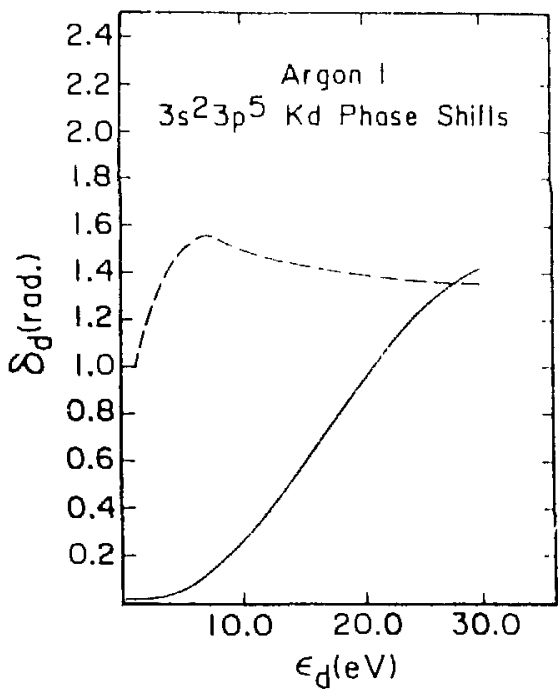

Fig. 17. Phase shifts, $\delta_{d}$, for the kd partial waves in neutral argon as a function of ejected-electron energy, $x_{d}$ (Ref. 39). Dashed curve, from a diatorted-wave calculation using the $\mathrm{CA}$ effective polential; solid curve, diatorted-wave calculation using the ' $\mathrm{p}$ effective potertial. 


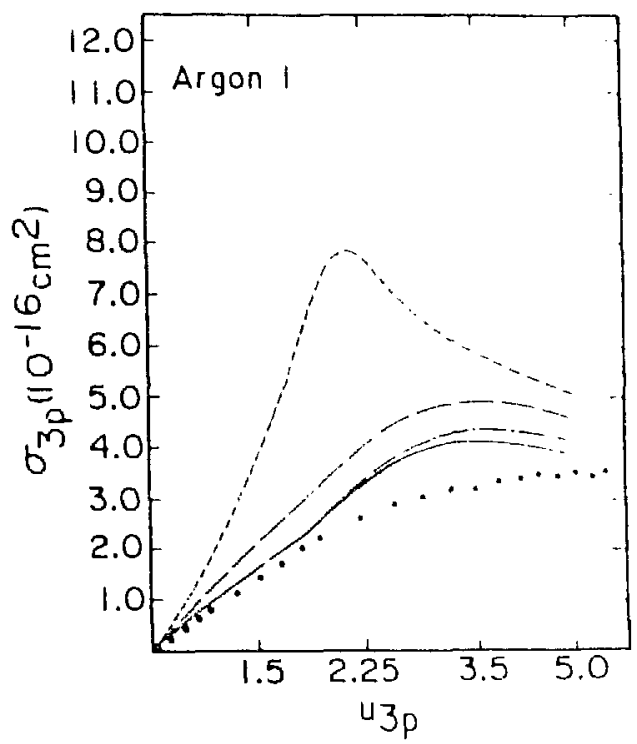

Fig. 18. 3p electron-impact ionization cross section, $\sigma_{3 p}$, of neutral argon (Ref. 39). The incident electron energy in throshhold units is represented by $u_{3}$. Short dash, caiculated uaing the $C A$ kd wave for the $3 p^{3} \mathrm{kd}$ ' $\mathrm{P}$ ejected-electron channel; long dash, calculated using the term-defendent kd wave for the $3 p^{3} k d^{1} P$ ejected-electron charınel; solid curve; term-dependent calculation with approximate ground-atate correlation; dot-dash, solid curve plus is subshall ionization; open circles, experimental measurementa (Ref. 4G).

of this isoelectronic sequence the effects of term dependence decrease rapidly with ionization stage. Not only is the barrier height diminished, reducing the difference between the $k d_{C A}$ and $k d{ }^{\prime}$ ' $p$ partial waves, but also, the relative importance of the $\mathrm{kd}$ ' $\mathrm{F}$ ' exit channel to the total ionization cross section decreases. 39

\section{CONCLUSIONS}

The sudden changes with atomic number in the binding energy of excited $d$ and $f$ electrons preceding the onset of transition and rareearth series of elements can be explained in terms of one-electron effective potentials. The change in binding energy and the associated wave function collepse are most abrupt when the potential contains a centrifugal barrier which is positive and well defined. Thus the contraction of wave functions is more gradual for d electrons than for $f$ electrons and occurs much less abruptly for ionized species than for neutrals.

Barriers in the effective potential for configurations of the type p" $d$ and $d^{\prime} f$ are enhanced by an unusually large positive exchange interaction for the ${ }^{1} \mathrm{P}$ terms. This may lead to a large difference between the ${ }^{2} P$ Hartree-Fock radial wave functions and the wave functions for all other terms of these configurations, especially near the beginning of the transition and rare-earth series of elements, where the collapse of the wave function is ebout to occur. This term dependence can have a significant influence on many atomic properties, including the excitation and ionization of both neutral and ionized species. 
The authors would like to thank $C$. Bottcher and S. Younger for a number of very useful discusaions. The collaborative offorts of the electron-ion beam experimental groups at ORNL and JILA are gratefully acknowledged. This wort was aupported by the Office of Fualon Energy, U. S. Department of Energy, under Contract No. DE-AC05-840R21400 with Martin Marietta Energy Systems, Inc., and under Contract No. DE-FG05-86ER53127 with Auburn University.

\section{REFERENCES}

1. E. Fermi, in: "Quantentheorie und Chemie," H. Falkenhagen, ed., S. Hinzel-Verlag, Leipzig, 1928, p. 95.

2. M. Mayer, Phye. Rev. 60, 184 (1941).

3. R. Latter, Phys. Rov. $\underline{99}, 510$ (1955).

4. A. R. P. Rau and U. Fano, Phys. Rev. 167, 7 (1968).

5. D. C. Griffin, K. L. Andrew, and R. D. Cowan, Phys. Rev. 177, 62 (1969).

6. D. C. Griffin, R. D. Cowan, and K. L. Andrew, Phys. Rev. A 3, 1233 (1971).

7. R. A. Falk, G. H. Dunn, D. C. Griffin, C. Bottcher, D. C. Gregory, D. H. Crandall, and M. S. Pindzola, Phys. Rev. Lett. 47, 494 (1981).

8. D. C. Griffin, C. Bottcher, ar,d M. S. Pindzola, Phys. Rev. A 25, 1374 (1982).

9. C. Bottcher, D. C. Griffin, and M. S. Pindzole, J. Phys. B 16, L65 (1983).

10. C. Froese Fischer, "The Hartree-Fock Method for Atoms," Wiley, New York (1977).

11. J. C. Slater, Phys. Rev. 81,385 (1951).

12. F. Herman and S. Skillman, "Atomic Structure Calculatione," Prentice-Hall, Inc., Englewood Cliffa, New Jersey (1963).

13. R. D. Cowan, Phys. Rev. 163, 54 (1967).

14. R. D. Cowan, "The Theory of Atomic Structure and Spectra," University of California Presa, Berkeley (1981).

15. J. P. Connerade, Contemp. Phys. 19, 415 (1978).

16. V. Kaufman and J. Sugar, J. Res. Natl. Bur. Stand. 71A, 583 (1967).

17. R. A. Roig and G. Tondello, J. Opt. Soc. Am. $\underline{65}, 829$ (1975).

18. R. D. Cowan, J. Opt. Soc. Am. 58, 924 (1958).

19. D. H. Hartree and W. Hartree, Proc. Roy. Soc. Al54, 588 (1936).

20. J. E. Hansen, J. Phys. B. $\underline{5}, 1083$ (1972).

21. D. C. Griffin and M. S. Pindzola, Comments At. Mol. Phys. 13, 1 (1983).

22. G. Wendin and A. F. Starace, J. Phys. B 11, 4119 (1978).

23. J. E. Hansen, A. W. Fliflet, and H. P. Kelly, J. Phys. B g, L127 (1975).

24. S. M. Younger, Phys. Rev. A 22, 2682 (1980).

25. G. Wendin, J. Phys. B 9, L297 (1976).

26. L. Brillouin, J. Phy8. Radium 3, 373 (1932).

27. M. S. Pindzola, D. C. Griffin, and C. Bottcher, Phys. Rev. A 27, 2331 (1983).

28. D. C. Gregory and A. M. Howald, Phys. Rev. A 34 (1986).

29. D. C. Griffin, C. Bottcher, M. S. Pindzola, S. M. Younger, D. C. Gregory, and D. H. Crandall, Phys. Rev. A 29, 1729 (1984).

30. D. C. Gregory and D. H. Crandall, Phys. Rev. A 27, 2338 (1983).

31. B. Pert and K. T. Dolder, J. Phys. B 1,872 (1968).

32. B. Pert, J. G. Stevenson, and K. T. Dolder, J. Phys. B 6, 146 (1973).

33. B. Pert and K. T. Dolder, J. Phys. B 8,56 (1975).

34. J. E. Hansen, J. Phys. B I, 1902 (1974). 
35. J. E. Hangen, J. Phys. B g, 2759 (1975).

36. P. G. Burke, A. E. Kingaton, and A. Thompoon, J. Phys. B 16 , L385 (1983).

37. D. C. Grifrin, M. S. Pindzola, and C. Bottcher, J. Phys. B 1?. 3183 (1984).

38. R. I. Karaziya, Sov. Phys. Usp. 24, 775 (1981).

39. S. M. Younger, Phys. Rev. A 26, 3177 (1982).

40. S. M. Younger, Submitted Phys. Rev. A (1986).

41. M. S. Pindzola, D. C. Griffin, and C. Bottcher, J. Phya. B 16, L355 (1983).

42. M. S. Pindzola, D. C. Griffin, C. Bottcher, D. H. Crandall,

R. A. Phaneuf, and D. C. Gregory, Phys. Rav. A. 29, 1749 (1984).

43. M. Gryzinski, Phy. Rov. 138, A336 (1965).

44. C. Achenbach, A. Muller, E. Salzborn, and R. Becker, Phys. Rev. LetL. 50, 2070 (1983).

45. S. M. Younger, private communication.

46. R. K. Aguni and M. V. Kurepa, J. Electron. Control 15, 41 (1963).

\section{DISCLAIMER}

This report was prepared as an account of work sponsored by an agency of the United States Governmerit. Neither the United States Government nor any agency thereof, nor any of their employees, makes any wirranty, express or implied, or assumes any legal liability or responsibility for the accuracy, completeness, or usefulness of any information, apparatus, product, or process disclused, ar represents that its use would not infringe privately owned rights. Reference herein to any specific commercial product, process, or service by trade name, trademark, manufacturer, or utherwise does not necessarily constitute or imply its endorsement, recommendation, or favoring by the United States Government or any agency thereof. The views and opirinno of authors expressed herein fo not necessarily stat... Hect those of the Uniteci States Government or any agency thereof. 